\title{
Electric Birefringence Imaging of Electrokinetic Agarose Orientation
}

\author{
MAUREEN LANAN, REED SHICK, * and MICHAEL D. MORRIS \\ Department of Chemistry, University of Michigan, Ann Arbor, Michigan 48109-1055
}

\begin{abstract}
SYNOPSIS
Time-resolved and steady-state electric birefringence imaging with a slow-scan video camera is used to study orientation during DNA agarose gel electrophoresis. The hydrodynamically induced gel distortion is shown to be the major source of birefringence under electrophoresis running conditions and to generate a birefringence image that approximates the image of the DNA concentration gradient in the electric field direction. A fluid kinematic model is presented to describe the spatial distribution of steady-state birefringence and is verified with fluorescence measurements of DNA distribution. The stress-optic coefficient of $1 \%$ agarose gel is measured by mechanical compression and used to evaluate the magnitude of the induced strain on the gel during electrophoresis.
\end{abstract}

\section{INTRODUCTION}

The study of DNA orientation dynamics in gels has achieved new urgency because of the relation to practical DNA agarose gel electrophoresis and its theoretical description. ${ }^{1-4}$ Transient electric birefringence (TEB),${ }^{5-8}$ linear dichroism (LD),${ }^{9-11} \mathrm{flu}$ orescence-detected linear dichroism (FDLD), ${ }^{1,12}$ and electric birefringence frequency dispersion ${ }^{13}$ have been used to study the alignment of DNA in gels. Time constants for DNA orientation in agarose have been obtained by TEB. ${ }^{7,8}$ Entanglement of DNA with the agarose matrix is believed to account for longer DNA time constants than those observed in free solution. ${ }^{14}$ This explanation is consistent with the elongation predicted by reptation theory.

DNA fragments have unimodal decay characteristics in solution at low field strengths. ${ }^{15}$ In agarose gels, however, DNA exhibits multiexponential decay with the number of characteristic times dependent upon the chain length and the gel concentration. ${ }^{8}$ This time constants also vary with applied field strength. For 170 kilobase pair ( $\mathrm{kbp}$ ) fragments in $2 \%$ agarose gels, ${ }^{11}$ both the fast $(0.5-5 \mathrm{~s})$ and the

\footnotetext{
* Present address: Dow Chemical USA, Midland, MI 48667. Biopolymers, Vol. 31, 1095-1104 (1991) (C) 1991 John Wiley \& Sons, Inc.

CCC 0006-3525/91/091095-10\$04.00
}

slow (14-115 s) decay time constants increase with decreasing field strength.

The alignment kinetics of large gel-confined DNA fragments in electric fields are complicated. ${ }^{6,10,12}$ Orientation has been studied by FDLD, ${ }^{12} \mathrm{LD},{ }^{10}$ and electric birefringence. ${ }^{6}$ When a voltage pulse is first applied, a maximum DNA alignment occurs, followed by a local minimum. Steady state orientation is achieved after a delay that depends on the chain length and the field strength. Holzwarth et al. ${ }^{1}$ find that the maximum orientation occurs in $2 \mathrm{~s}$ for 48 $\mathrm{kbp}$ DNA at $10 \mathrm{~V} / \mathrm{cm}$ in $1 \%$ agarose. The overshoot in DNA alignment has been attributed to the Rouse chain stretching and compression of the DNA. ${ }^{1,4}$

At least two types of agarose alignment accompany electrophoresis. One is independent of the presence of $\mathrm{DNA}^{6,16}$ and the other is associated with DNA bands. ${ }^{10}$ Pure agarose gels follow Kerr's law in low electric fields. ${ }^{16}$ The gel birefringence at fields below $\sim 100 \mathrm{~V} / \mathrm{cm}$ has been found to have either positive or negative birefringence. The birefringence sign for a particular gel inverts when the field polarity is reversed. The same phenomenon is observed in unresolved DNA-agarose mixtures. ${ }^{6}$ Bulk agarose orientation is a slow process with a time constant of approximately $10 \mathrm{~s},{ }^{16}$ the same order of magnitude as the slow relaxation times for large gel-confined DNA fragments at moderate field strengths.

Stronger agarose orientation with a determinate 
sign is observed in gel regions containing DNA. This was first observed by Jonsson et al., ${ }^{10}$ using anisotropic light scattering at $320 \mathrm{~nm}$. The effect was attributed to DNA-induced electro-osmotic flow, which creates a buffer volume gradient across the DNA band. In response to the force caused by this gradient, the gel becomes extended in the direction of cation electrophoretic transport. The gel is compressed in the opposite direction, which is the direction of DNA migration.

Although the source of induced gel distortion is known, there have been no published measurements of the magnitude of this effect, and no attempt to test the influence of localized gel orientation on DNA migration and dynamics. The nucleic acid must work against the distorted gel, whose orientation effects migration of fragments. ${ }^{17}$ In this paper we develop a fluid-kinematic model for induced gel distortion and its effect on gel electric birefringence. This model permits us to measure the magnitude of the restoring force using photoelastic stress analysis techniques and to relate these measurements to the applied electromotive force.

Our measurements are made in slab gels containing separated Hind III fragments and employ slowscan video imaging measurements of spatially resolved electric birefringence. Solid-state video cameras have been used as imaging densitometers to detect ethidium bromide stained DNA in agarose gels.$^{18}$ Fluorescence video microscopy has been used to observe individual DNA molecules migrating through agarose ${ }^{19,20}$ However, video techniques have apparently not been used to monitor gel electric birefringence.

A slow-scan video CCD camera has useful properties for electric birefringence measurements. The camera has excellent sensitivity and a wide-dynamic range, which allows detection of small birefringence changes. ${ }^{21}$ Imaging detection is inherently faster than single-point measurements for investigation of alignment of multiple DNA fragment bands. As we shall show, imaging clearly demonstrates the spatial relationship between gel distortion and nucleic acid concentration. However, at $0.5-5$ frames $/ \mathrm{s}$, imaging with the typical slow-scan video camera does not permit investigation of dynamic properties on a time scale of less than about $1 \mathrm{~s}$.

The charge per base pair of DNA entangled in an agarose gel has been estimated using measurements of fragment elongation ${ }^{22}$ in an electric field to be $0.06-0.10$. In free solution, electrophoretic mobility ${ }^{23}$ yields $0.4-0.8$ electrons per base pair, depending on ionic strength. Values determined from elongation measurements include implicit correc- tions for opposing electrokinetic and viscous forces ${ }^{22}$ in solution that are neglected in mobility measurements. The effective charge per base pair from elongation measurements is used in the present work to compare the magnitude of the stress from the electric field on the fragments to the mechanical gel distortion observed experimentally.

\section{THEORY}

By applying an electric field to an anisotropic charged molecule, linear birefringence can be induced. At low field strengths, Kerr law relates the induced birefringence to the field strength. Stated in terms of optical retardation $\delta$, Kerr's law ${ }^{24}$ is

$$
\delta=\frac{2 \pi d}{\lambda} n C_{\mathrm{v}} K_{\mathrm{sp}} E^{2}
$$

where $\lambda$ is the wavelength of light used, $d$ is the path length, $n$ is the isotropic refractive index, $C_{\mathrm{v}}$ is the concentration of DNA given in grams per unit volume times the partial molar volume $\left(\mathrm{cm}^{3} / \mathrm{g}\right), K_{\mathrm{sp}}$ $\left(\mathrm{V}^{-2} / \mathrm{cm}^{2}\right)$ is the specific Kerr constant, and $E(\mathrm{~V} /$ $\mathrm{cm}$ ) is the field strength.

Electric birefringence is commonly measured by monitoring the intensity change through two linear polarizers set with their transmission axes at $45^{\circ}$ with respect to the aligning voltage. A quarter wave plate is positioned with its slow axis parallel to the transmission axis of one of the polarizers. The analyzer is then turned through an angle $\alpha$ away from the crossed position. This configuration optimizes sensitivity and distinguishes positive and negative birefringence.

The intensity changes measured in this configuration ${ }^{24}$ are related to the optical retardance of the sample. For small $\delta$ :

$$
\delta=\frac{\Delta I_{\delta}}{I_{\alpha}-I_{\mathrm{SL}}}\left[\frac{2 \sin ^{2}\left(\alpha+\frac{\delta_{0}}{2}\right)}{\sin \left(2 \alpha+\delta_{0}\right)}\right]
$$

where $\Delta I_{\delta}$ is the difference in birefringence intensity between the field-on and field-off conditions, $I_{\alpha}$ is the average intensity at the analyzer angle $\alpha, I_{\mathrm{SL}}$ is the stray light intensity with crossed polarizers, and $\delta_{0}$ is the optical retardance of the cell.

For a photoelastic material, such as agarose, optical retardance is directly related to the applied stress through the stress optic law ${ }^{25}$ :

$$
\delta=Z d \Delta \sigma
$$


where $Z$ is the stress-optic coefficient, $d$ is the path length through the material, and $\Delta \sigma$ is the applied stress. Using this convention, the sign of the birefringence changes when the direction of the applied stress reverses.

The deviatoric stress equation relates velocity to stress in a flowing medium. ${ }^{26}$

$$
\Delta \sigma=\eta\left(\frac{\partial v_{x}}{\partial y}+\frac{\partial v_{\mathrm{y}}}{\partial x}\right)
$$

The $\eta$ is the shear viscosity, and $v_{i}$ is the flow velocity in direction $i$. If we assume that the buffer flow velocity is goverend by electroosmosis, then

$$
v \propto q E
$$

Assuming that the agarose molecules are nonionic, $q$ is directly proportional to the DNA concentration. Electroosmosis causes a localized stress, which varies as

$$
\Delta \sigma=\frac{K E}{A}\left[\frac{\partial C^{\mathrm{DNA}}(x)}{\partial x}\right]
$$

where $K$ is the scaling factor between charge and molecular concentration $C(x)$, and $A$ is the crosssectional area. This equation assumes $C(x)$ is not a function of $y$.

In the limit of an incompressible fluid, the electrokinetic equation for stress applies. ${ }^{27}$

$$
\Delta \sigma=\eta \nabla^{2} v-\frac{\partial p}{\partial x}-\sum_{i=0}^{j} n_{i} z_{i} e \frac{\partial \Psi}{\partial x}
$$

In Eq. (6), $\eta$ is shear viscosity, $v$ is the bulk fluid velocity, $p$ is pressure, $n_{i}$ is the number density of particles, $z$ is the valence of the ion, $e$ is the charge denisty, and $\delta \Psi / \delta x$ is the induced electric field gradient. The terms on the right-hand side of Eq. (7) represent viscous drag, local pessure gradients, and electrical forces, respectively, per unit volume. The $\delta p / \delta x$ is the only first-order term in which the derivative depends directly on the polyelectrolyte spatial derivative. By analogy to the incompressible fluid, the major induced stresses in electrophoresis can be attributed to hydrodynamic pressure gradients. $^{10}$

To a first approximation, the total birefringence at any given time is the linear combination of the Kerr response of DNA, the Kerr response of gel, and the localized mechanical distortion of the gel. Using the Kerr law, [Eq. (2)] and Eq. (6):

$$
\begin{aligned}
\delta(x, E) & =\frac{2 \pi d}{\lambda} n C_{\mathrm{v}}^{\mathrm{DNA}}(x) K_{\mathrm{sp}}^{\mathrm{DNA}} E^{2}+\frac{2 \pi d}{\lambda} n C_{\mathrm{v}}^{\mathrm{gel}} \\
& \times K_{\mathrm{sp}}^{\mathrm{gel}} E^{2}+\frac{Z(t) d K E}{A}\left\{\frac{\partial\left[C_{\mathrm{v}}^{\mathrm{DNA}}(x)\right]}{\partial(x)}\right\}
\end{aligned}
$$

Here, the stress-topic coefficient is written as a function of time, $Z(t)$.

The electromotive stress acting on a nucleic acid sample during electrophoresis can be written using the definition of electromotive force ${ }^{28}$ and the definition of stress ${ }^{22}$ as

$$
\Delta \sigma_{\mathrm{el}}=\frac{q E}{A}
$$

The charge $q$ is the product of the number of DNA base pairs present and the average charge per base pair. Strictly, the stress is a tensor quantity. However, it is possible to approximate the stress tensor by assuming the DNA to be homogeneously distributed across the width and the depth of bands, perpendicular to the migration direction.

\section{EXPERIMENTAL}

\section{Reagents}

GTG grade Sea Plaque agarose (FMC Bioproducts) was dissolved in TAE buffer ( $40 \mathrm{~m} M$ Tris acetate, $1 \mathrm{~m} M$ EDTA, $\mathrm{pH}$ 8) using a stirring hot plate following standard protocol. ${ }^{29}$ The final gel concentration was $1 \%$. Phage lambda Hind III (BRL) was stored in aliquots at $-30^{\circ} \mathrm{C}$. A fresh aliquot was used for each experiment. Samples were diluted in fragment buffer ( $20 \mathrm{~m} M$ Tris $\mathrm{HCl}, 20 \mathrm{~m} \mathrm{M} \mathrm{NaCl}, 0.1$ $\mathrm{m} M$ EDTA, $\mathrm{pH} 8$ ) and heated to $65^{\circ} \mathrm{C}$ for $10 \mathrm{~min}$. The samples were diluted to $4-40 \mathrm{ng} / \mu \mathrm{L}$, depending upon the sample loading, in a final buffer concentration of $1 \times$ TAE.

The horizontal electrophoresis cell was similar to the design of Schaffner ${ }^{30}$ except that the bottom of the chamber was an optical quality $7 \times 10 \mathrm{~cm}$ Pyrex glass plate. A $1 \times .05 \mathrm{~cm}$ PVC tube (ID $0.3 \mathrm{~cm}$ ) was glued to the glass plate to provide a gel-free region, which was monitored to compensate for light source intensity fluctuations. Gels were cast directly on the plate.

Agarose $(20 \mathrm{~mL})$ was cast at $65^{\circ} \mathrm{C}$ into the cell with $1 \times 5.2 \mathrm{~mm}$ sample wells held $1 \mathrm{~mm}$ above the 
glass. The gels were covered with buffer after $30 \mathrm{~min}$ and maintained at room temperature before use. For optimal sensitivity, gels were allowed to set overnight in buffer at room temperature. In some experiments Velcro strips on the cell bottom prevented the gel from moving.

To load DNA samples, buffer was removed from the cell until it no longer covered the gel. In addition, sample wells were drained before loading. The wells were loaded with $10-15 \mu \mathrm{L}$ of sample and a field of about $15 \mathrm{~V} / \mathrm{cm}$ was applied for about $1 \mathrm{~min}$ to move the fragments into the gel. Then $1 \times$ TAE was added to give a final volume of $170 \mathrm{~mL}$ in the cell. This volume was sufficient to cover the gel with $1-2 \mathrm{~mm}$ of buffer. Electrophoresis was performed at 3-5 V/ $\mathrm{cm}$ for up to $2 \mathrm{~h}$ using a BioRad model $250 / 2.5$ power supply.

Fluorescence staining was carried out in the cell by adding ethidium bromide to give $0.5 \mu \mathrm{g} / \mathrm{mL}$ and stirring gently. The excess ethidium was left in place during visualization. Fluoresence was excited at 300 $\mathrm{nm}$ and recorded through a red cutoff filter (Nikon R60), which passed wavelengths longer than about $580 \mathrm{~nm}$.

\section{Equipment}

The birefringence photometer (Figure 1) consisted of a light source, polarizing optics, electrophoresis cell, imaging optics, and video camera. A Dell 310 computer (20-MHz 80386, MS-DOS 3.2) provided voltage pulse control through a transistor switch and camera control. A Metrabyte DASH-16 A/D board with an independent crystal clock provided precise timing.

A halogen $90 \mathrm{~W}$ flood light, with its brightest area masked, illuminated the sample through a diffuser and a 4-inch square linear plastic film polarizer (Polaroid NH32 or NH38s) mounted with the fast axis $45^{\circ}$ to the electric field direction. A commercial quality 2-inch diameter, AR-coated quarter-wave plate $(650 \mathrm{~nm}, 0.2504$ waves, Meadowlark Optics) and second linear polarizer were mounted in rotation stages, accurate to within $1^{\circ}$. A $40-\mathrm{nm}$ bandpass filter (Corion S40-650-S-M327) centered at $650 \mathrm{~nm}$ defined the wavelength region observed. The polarizing optics were aligned as described previously ${ }^{24}$ to give positive signals (increased transmission) for negative birefringence. The analyzer was rotated to compensate for stray light. In most images the background intensity measured was used to compensate for light source fluctuations.

Either a Nikon $50 \mathrm{~mm}$ f.1.f $/ 2.8$ or a Nikon MicroNikkor $60 \mathrm{~mm} \mathrm{f} / 2.8$ lens were used with a PK-11A 8-mm extension tube, to provide additional magnification. A Photometrics Star 1 camera equipped with a Thompson 7883 chip operated at $-45^{\circ} \mathrm{C}$ and a 12 bit A/D converter was used to record images. The camera was focused and distances were calibrated by recording the image of an USAF-1951 resolution target placed in the plane of the gel.

The field strength was measured directly with a $>10 \mathrm{M} \Omega$ input impedance digital multimeter (Omega) connected to fixed platinum probes placed in the gel. Temperature was measured with an Omega HH72 thermocouple thermometer, using a

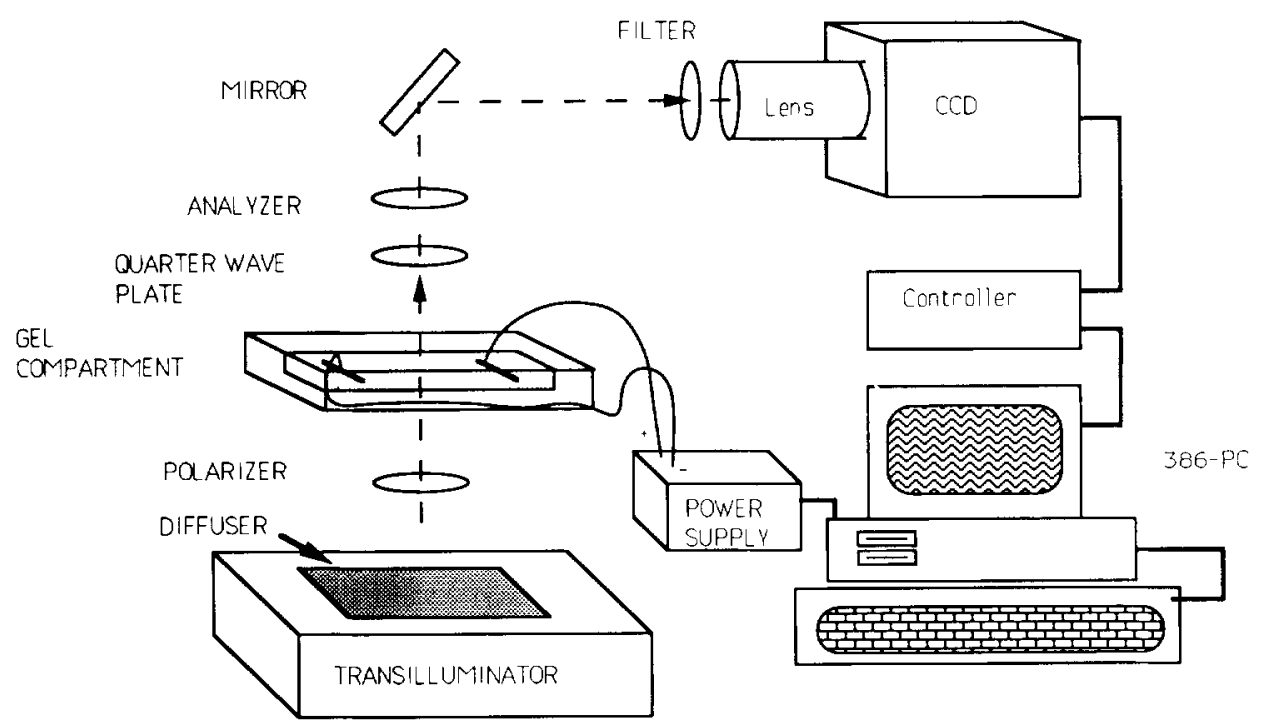

Figure 1. Electric birefringence imaging system. 
type $\mathrm{K}$ thermocouple sealed into a Pasteur pipet filled with thermally conductive paste.

\section{Procedure}

Electric Birefringence. After electrophoresis, the voltage was removed for approximately $30 \mathrm{~min}$. The time response of electric birefringence was then measured using an electric field, which was applied to the gel for $14 \mathrm{~s}$. Images were recorded every $5 \mathrm{~s}$ ( $6 \mathrm{~s}$ in some sequences) for $120 \mathrm{~s}$ from the beginning of the pulse.

Voltage dependence of electric birefringence was measured during the last $1-3 \mathrm{~s}$ of a 14-s electric field pulse. A background image was recorded at the end of a 25-s field-free relaxation time. A 14-s pulse with opposite polarity was then applied and the birefringence response recorded again to establish the response to an electric field inversion.

For calibration, the incident intensity was taken to be the average intensity in the gel-free area. To correct for local variations in DNA content across the width of a band, calculations were made on the average intensity perpendicular to the migration direction. These transverse averages were then normalized to the appropriate average incident intensity. The values were used in calculations based on Eq. (2). With this method, photometry had a between-image standard deviation of $\pm 0.25 \%$.

To obtain viewable electric birefringence images on a video monitor, care was taken to minimize dynamic range limitations caused by processing 12 -bit images through the 8-bit Macintosh computer image processing program, IMAGE. ${ }^{31}$ Images were compensated and background subtracted with 12 -bit accuracy. The difference values were clipped to 9 bits centered at 0 and then scaled to 8 bits $(0-255)$. An offset was added to give zero intensity a value of 127 on this scale. At a cost of reducing the dynamic range by a factor of 2 , the procedure yielded images viewable with conventional video hardware and driver software.

For some calculations transverse average plots were also obtained using, IMAGE. ${ }^{31}$ After preforming a median smooth on the image, transverse averages were generated. These averages were used to obtain relative peak areas following IMAGE protocols. Data was numerically differentiated or integrated in ASYSTANT (Keithley Software).

Stress-Optic Response. The stress-optic response was measured using mechanical compression of the gel. SeaPlaque agarose $(1 \%)$ was cast in a $1-\mathrm{cm}$ path-length quartz spectrophotometer cell. Known stresses were applied by compressing the gel with a square plunger that supported 1-20-g laboratory balance weights. Correction was made for the 2.66 $\mathrm{g}$ mass of the plunger.

The birefringence sign for compression and extension was determined by applying mechanical stresses to gels, using a Delrin block imbedded in the agarose. The Delrin block $(22.8 \times 4.8 \times 3.1 \mathrm{~mm})$ was tied to a thin thread through $1.5-\mathrm{mm}$ diameter holes. The block was placed on a 3 inch $\times 1$ inch microscope slide and $16 \mathrm{~mL}$ of $1 \%$ SeaPlaque in $1 \times$ TAE was cast around it. The microscope slide was mounted in the birefringence imaging instrument. Images were recorded with stresses applied with 120 -g weights attached to the thread.

\section{RESULTS AND DISCUSSION}

\section{Photo-elastic Stress Measurements}

The stress-optic coefficient was measured in compression for three samples of the same $1 \%$ agarose gel batch, freshly prepared and aged for up to 2 days. In all experiments, compression of the gel caused negative birefringence. As shown in Table $I$, the stress-optic coefficient increases as the gel ages, although the change is greatest during the first day. These results are consistent with other observations that the structure of agarose continues to develop as the gel ages. ${ }^{16}$ For different aliquots of the same gel of the same age, the stress-optic coefficient is reproducible within experimental error $(\leq 10 \%)$. The standard deviation for measurements on three preparations of the same nominal gel composition is $36 \%$.

In a photoelastic material, the sign of the birefringence changes if the direction of the applied stress is reversed. In a material subjected to both extension and compression, both positive and negative birefringence would be observed. We tested this prediction for an agarose gel using calibrated

Table I Stress-Optic Coefficient of $1 \%$ SeaPlaque Agarose Gel

\begin{tabular}{rcrrr}
\hline Gel & $\begin{array}{c}\text { Age } \\
\text { (day) }\end{array}$ & $\begin{array}{c}\text { SOC } 10^{5} \\
\left({ }^{\circ} / \mathrm{Pa} \mathrm{cm}\right)\end{array}$ & \multicolumn{1}{c}{$n$} & \multicolumn{1}{c}{$r^{2}$} \\
\hline \multirow{2}{*}{1} & 0 & $3.4 \pm 0.2$ & 8 & 0.8046 \\
& 0 & $2.8 \pm 0.4$ & 20 & 0.9099 \\
& 2 & $30.3 \pm 2.1$ & 20 & 0.9444 \\
2 & 1 & $24.6 \pm 1.9$ & 20 & 0.8921 \\
3 & 1 & $23.9 \pm 0.8$ & 20 & 0.9753 \\
\hline
\end{tabular}


stresses applied to a beam imbedded in the gel, using our video system. In the direction of compression, the birefringence was negative, in agreement with compression results obtained in a spectrophotometer cell. In the direction of extension, the birefringence was positive. The stress-optic coefficient was approximately $5.4 \times 10^{-5}\left({ }^{\circ} / \mathrm{Pa} \mathrm{cm}\right)$, in reasonably good agreement with our spectrophotometer cell for a gel tested the same day it was cast. We view these results as qualitative verification of the stress-optic properties of agarose gels. The video instrument design does not allow easy acquisition of quantitative stress-optic data.

\section{Steady-State Electric Birefringence Imaging}

The large field of view in the imaging system makes crystal polarizing optics impractical. We have found that nonlaminated plastic film Polaroid gives relatively uniform polarization with a stray light constant of $2 \times 10^{-4}$. This value is about $4 \times$ worse than the $5 \times 10^{-5}$ typically observed ${ }^{5,7}$ for Glan-Thompson prisms. The optimal signal to noise was obtained with $\alpha=6 \pm 1^{\circ}$, in agreement with theoretical ${ }^{32}$ predictions for this stray light level.

Figure 2 shows a typical electric birefringence image signal from a single gel lane. The blank re- gions of gel are grey. Each DNA fragment band produces negative birefringence, shown as a white region, on the anodic side of the band and positive birefringence, a black region, on the cathodic side.

Figure 2 illustrates the characteristic two-lobe response of each fragment, although the lobes are difficult to observe for the weak $2.2 \mathrm{kbp}$ response. Negative birefringence is always observed on the anodic side of the bands, as comparison of the upper and lower images demonstrates. As we show with field-strength dependence data below, the two-lobe response arises entirely from the agarose, not the DNA, and is consistent with compression of the gel on the anodic side and extension on the cathodic side. Our observations confirm the DNA-dependent gel orientation reported by Jonsson et al. ${ }^{10}$ The implication is that the gel double helices are aligned parallel to the migration direction of the DNA on the trailing side of a DNA band and perpendicular to the DNA migration direction on the leading edge. As Jonsson et al., ${ }^{10}$ have suggested, the gel compresses in response to increasing buffer flow into it on the leading edge of the band and extends on the trailing edge.

For comparison, we show transverse average plots of the same gel lane in Figure 3. The figure also shows a transverse average plot of the ethidium flu-

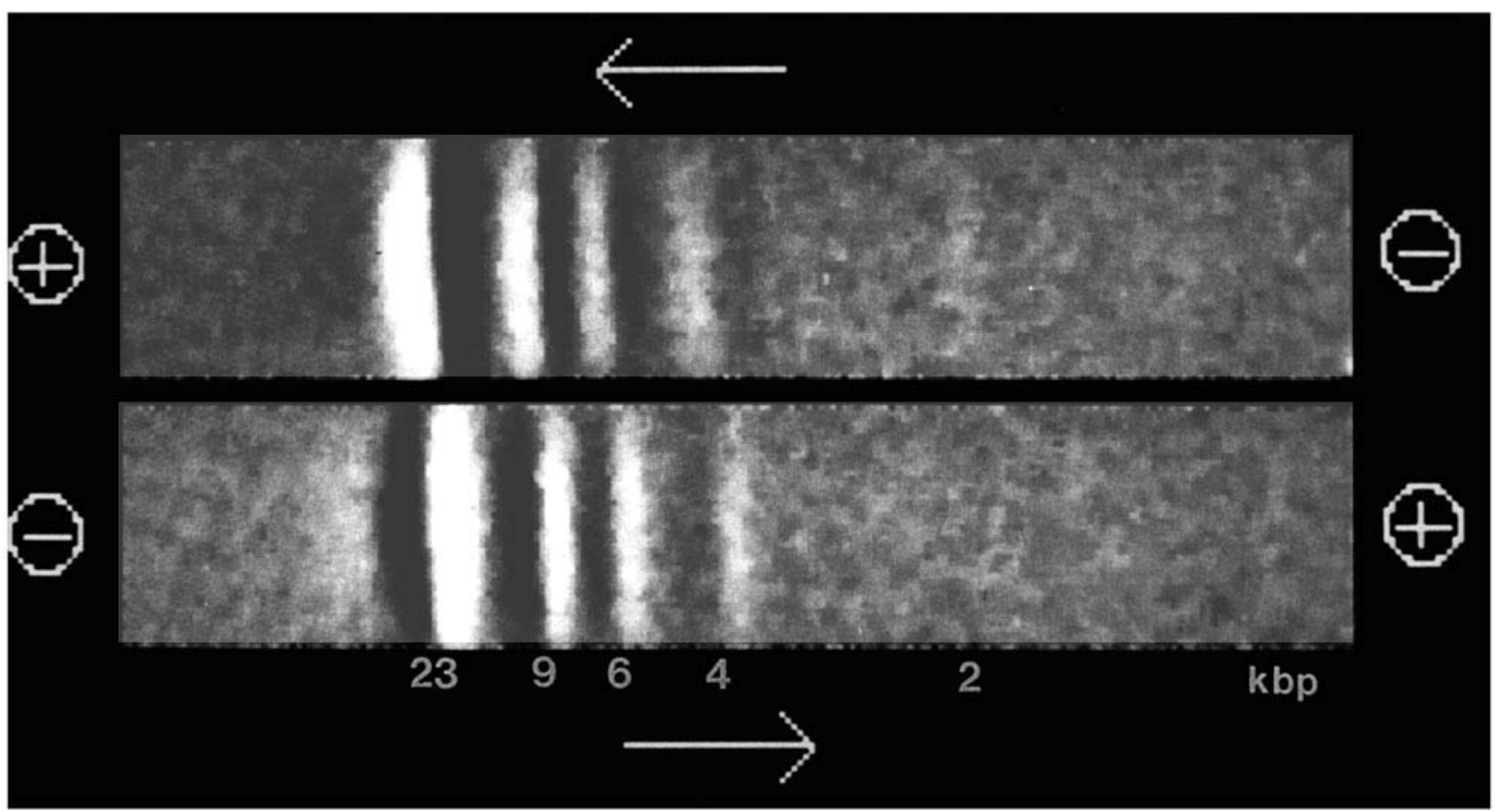

Figure 2. Electric birefringence image gel containing $500 \mathrm{ng}$ total loading Hind III DNA fragments in $1 \%$ agarose, $1 \times$ TAE buffer $10 \mathrm{sec}$ after imposition of a $6.0 \mathrm{~V} / \mathrm{cm}$ voltage pulse; $1.6 \mathrm{sec}$ integration time. The electric field direction is as shown by the arrows. 


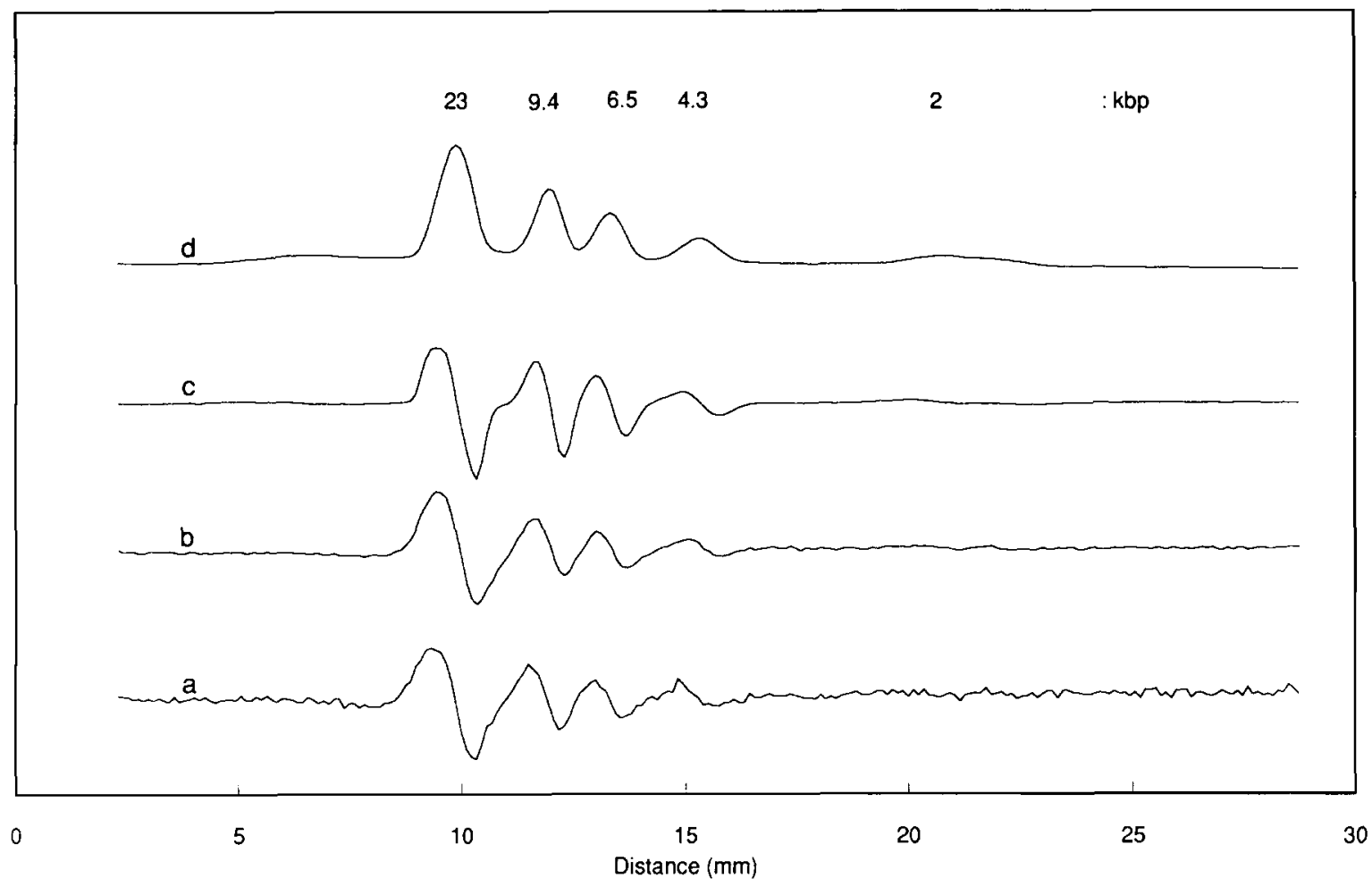

Figure 3. Comparison of electric birefringence imaging transverse averages and fluorescence intensity for $500 \mathrm{ng}$ total loading of $\mathrm{H}$ ind III DNA fragments in $1 \%$ agarose, $1 \times$ TAE buffer $10 \mathrm{sec}$ after imposition of (a) $2.25 \mathrm{~V} / \mathrm{cm}$ or (b) $8.19 \mathrm{~V} / \mathrm{cm}$ voltage pulse; 1.6 sec integration time. (c) Derivative of the fluorescence intensity. (d) Fluorescence intensity.

orescence of the lane, as well as the spatial derivative of this fluorescence plot. Visual inspection shows the similarity between the fluorescence spatial derivative and the observed electric birefringence. This congruence is observed at fields as high as $8.2 \mathrm{~V} / \mathrm{cm}$.

\section{Transient Electric Birefringence Imaging}

To verify that our images are taken under steadystate or near-steady state conditions, we have measured the evolution and decay of birefringence, within the resolution limitations (seconds) of our imaging system. The birefringence decay (peak maximum to peak minimum) for 6-, 9-, and $23-\mathrm{kbp}$ DNA after a $10-\mathrm{s}, 20-\mathrm{V} / \mathrm{cm}$ electric field pulse yields a decay time constant of approximately $10 \mathrm{~s}$ for the $23-\mathrm{kbp}$ fragment. The shorter fragments have time constants too short for us to measure, as expected. At $5 \mathrm{~V} / \mathrm{cm}$ the time constants are slightly longer. Rise times are similar. The time constant for decay of agarose alone is about $14 \mathrm{~s}$. To allow the gel orientation background to decay to negligible levels, blank images were recorded after the voltage had been removed for at least $25 \mathrm{~s}$ in all imaging experiments reported here.

The choice of a 25-s decay time to define a birefringence-free signal only holds for the maximum field strengths used in this work. However, even at $5 \mathrm{~V} / \mathrm{cm}$ the birefringence decay is nearly complete after $25 \mathrm{~s}$ for both the gel blank signal and all of the DNA-dependent signals. In most experiments, we measured birefringence images $14 \mathrm{~s}$ after application of the electric field. At this time the induced gel response is nearly complete, as measured by the decay of two-lobed signals in the DNA-containing regions. Although true steady-state response is not observed, the short pulse duration reduces artifacts from Joule heating at high electric fields.

The different time dependencies of contributions to the birefringence signal is shown clearly in Figure 4. Here, an overloaded ( $1 \mu \mathrm{g}$ ) gel is used to emphasize the Kerr response of the 23-kbp fragment at short times. After imposition of a voltage pulse, the spatial distribution evolves from almost peak shaped at $5 \mathrm{~s}$ to the two-lobed steady-state behavior at $15 \mathrm{~s}$. When the voltage pulse is removed, the response evolves back towards the time-zero background. A similar 


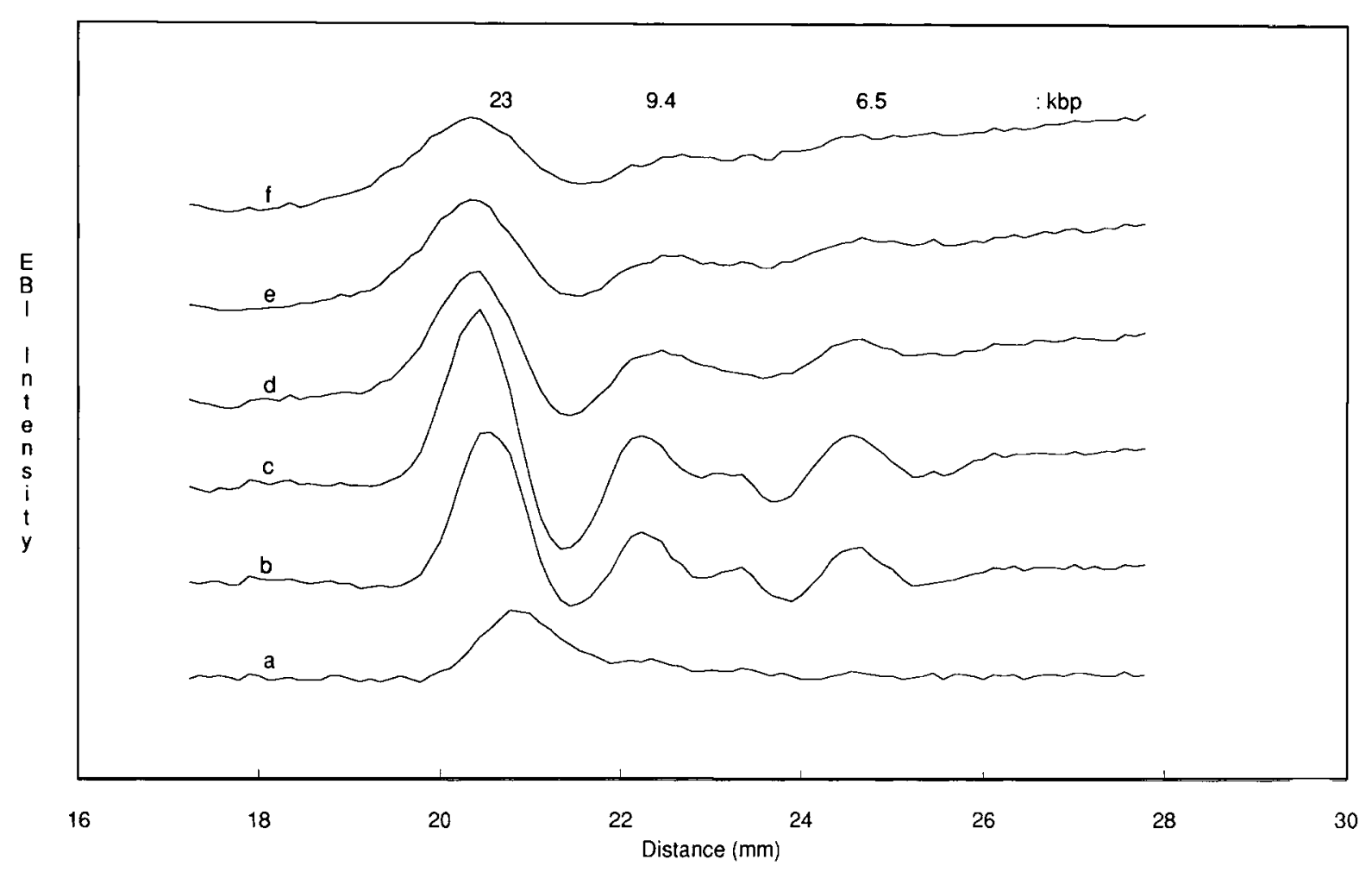

Figure 4. Growth and decay of electric birefringence intensity of gel containing $1 \mu \mathrm{g}$ Hind III DNA fragments in $1 \%$ agarose, $1 \times$ TAE buffer during imposition and after removal of a $20 \mathrm{~V} / \mathrm{cm}$ pulse. Integration time, $1.0 \mathrm{sec}$. (a) $5 \mathrm{sec}$ after start of pulse. (b) $10 \mathrm{sec}$ after start of pulse. (c) $15 \mathrm{sec}$ after start of pulse. (d) at time of pulse removal (open circuit). (e) $5 \mathrm{sec}$ after open circuit. (f) $10 \mathrm{sec}$ after open circuit.

time course has been reported by Jonsson et al. ${ }^{10}$ They find that 2.3-kbp DNA and 23-kbp DNA align in $\sim 0.1 \mathrm{~s}$ while agarose aligns more slowly. They observed that agarose orientation occurs in about 1 $\mathrm{s}$ for the $2.3 \mathrm{~kb}$ fragment and in $6-12 \mathrm{~s}$ for the $23-$ kbp fragments, depending on the previous voltage pulse history of the gel.

The electric field dependence of birefringence distinguishes classical (Kerr) orientation of either a DNA fragment or of agarose itself from the induced gel distortion. We have measured the field dependence of the electric birefringence response of three different batches of $1 \%$ agarose over the range of 2 $22 \mathrm{~V} / \mathrm{cm}$. The average slope of a log-log plot of optical retardation vs field strength is $2.2 \pm 0.3$. Thus, in the absence of DNA agarose follows the quadratic field strength dependence of the Kerr law.

The behavior is entirely different in regions of the gel containing nucleic acid fragments. Using data for three different $1 \%$ agarose gels with loadings from $20-250 \mathrm{ng}$, and using data for the 23-, 9-, 6-, and 4-kbp bands, the steady-state peak-peak response over the field range of $2-22 \mathrm{~V} / \mathrm{cm}$ could be fitted to a linear equation, with a correlation coefficient squared $\left(r^{2}\right)$ of 0.98 . The same linear response is observed for the area under the peak, as measured in IMAGE $\left(r^{2}=0.97\right)$. The DNA signals in these gels have the two-lobed, or derivative response, shown in Figures 2 and 3. This non-Kerr behavior is exactly as predicted for electrokinetic gel distortion, as described by Eq. (6). A similar linear voltage dependence for induced gel orientation is reported by Jonsson et al. ${ }^{10}$

From both its spatial dependence and electric field dependence, we conclude that the major contribution to the steady-state electric birefringence under electrophoresis running conditions is gel distortion. Unless the gel is drastically overloaded or the running voltage is unrealistically high, the contribution from the Kerr response of the nucleic acid itself is too small to be measured by our apparatus.

\section{DNA Concentration Dependence of the Steady-State Birefringence}

The peak area under the birefringence signal is linear $(r=0.98)$ with DNA fragment loading for all 
fragment sizes (2.2-23 kbp) from 20-250 ng. With the present system the limit of detection $(\mathrm{S} / \mathrm{N}=2)$ is approximately $20 \mathrm{ng}$ DNA at an electric field strength of $8.2 \mathrm{~V} / \mathrm{cm}$. The present instrument provides good results with the 100-200-ng loadings commonly used for restriction fragment separations and other electrophoretic separations. It can function as a photometer that requires no staining/destaining and does not need UV light for operation. Further investigations of the analytical utility of this technique will be presented in a separate communication.

\section{Comparison of Mechanical Stress to EMF Stress}

With the measured stress-optic coefficient for agarose, we can use Eq. 3 to compare the gel strain we observe to the calculated effect of an electromotive force acting on a DNA band. Table II summarizes the fraction of the total stress from a DNA band apparently producing gel distortion. Results are presented for a range of charge per base pair values taken from the literature for buffers with similar ionic strength to TAE. The results are averaged for four fragment lengths, at two total loadings, and three different field strengths. The weight of sample per band is calculated from the manufacturer's concentration measurement. The band area is estimated to be $0.2 \times 0.52 \mathrm{~cm}$.

As Table II shows, the electrokinetically induced distortion of the gel is of the same order of magnitude as the net, calculated EMF per unit area using the smallest value for the charge per base pair. Using the free solution value ${ }^{23}$ of 0.4 electrons per base pair instead gives an average fraction of $0.16 \pm 0.02$ of the total EMF. These calculations should be viewed as qualitative only. The Smith and Bendich ${ }^{22}$ estimate of the plasmid charge per base pair may

Table II Fraction of Theoretical Stress Observed as Gel Distortion"

\begin{tabular}{cc}
$\begin{array}{c}\text { Charge per Base Pair } \\
(\mathrm{e} / \mathrm{bp})\end{array}$ & $\Delta \sigma_{\text {experimental }} / \Delta \sigma_{\text {theoretical }}$ \\
\hline 0.06 & $1.1 \pm 0.2$ \\
0.1 & $0.6 \pm 0.1$ \\
0.4 & $0.16 \pm 0.03$ \\
0.8 & $0.08 \pm 0.01$ \\
\hline
\end{tabular}

\footnotetext{
- Values represent the average and standard deviation for 13 measurements. We use data for 23-, 9-, 6-, and 4-kbp DNA at $2.55,6.06$, and $8.19 \mathrm{~V} / \mathrm{cm}$, where bands contain $97.3,67.8,45.1$, and $47.8 \mathrm{ng}$. A band containing $19.5 \mathrm{ng}$ was used only at $8.19 \mathrm{~V} /$ $\mathrm{cm}$.
}

not apply to our short fragment lengths, different ionic strength, and more dilute gel. Use of the average DNA concentration in a band is another possible source of systematic error since it neglects the effects of band spreading.

Our calculations predict an apparent physical impossibly when 0.06 electrons per base pair is used. The fraction of the applied EMF that generates gel distortion exceeds unity. If the birefringence measurements are qualitatively correct, this may result from use of an inappropriate charge per base pair parameter. The Smith and Bendich ${ }^{22}$ values describe only the net force responsible for elastic stretching of DNA plasmids. The free solution values, however, correct for less of the viscous and electrokinetic processes associated with completely elongated DNA fragments. Since the gel is distorted by electro-osmotic flow, neglecting parameters that produce hydrodynamic flow would increase the relative significance of gel distortion. Perhaps the force required to distort the gel is the same order of magnitude as the force responsible for DNA chain elongation, but much less when compared to the total stresses produced by a fragment band in an electric field.

Despite these uncertainties, our data strongly suggest that mechanical distortion of the gel cannot be neglected in theoretical discriptions of nucleic acid electrophoresis. The gel is not a passive matrix, but an elastic medium whose compression and extension can affect nucleic acid motions. The effects should be especially pronounced in field inversion, pulsed field, or other electrophoretic techniques in which the electric field is periodically varied. Agarose that has its pores aligned by being formed in an electric field has a different mobility for DNA fragments than gel that has not been aligned. ${ }^{17}$ The interplay between electrokinetic orientation dynamics and nucleic acid dynamics may result in the peculiar transient behavior that accompanies changes in field magnitude or direction.

\section{CONCLUSION}

Because it permits simultaneous spatial and temporal investigations, electric birefringence imaging is a useful technique for studying orientation dynamics in DNA-matrix systems. Gradient effects, including induced agarose orientation, are more easily studied than with single-point measurement systems. Our results show that the magnitude of the gel distortion can be conveniently measured and related, at least qualitatively, to the expected effects of the applied electromotive force. Indeed, our mea- 
surements confirm that the interaction between gel and DNA cannot be ignored under ordinary electrophoretic conditions.

Equally significant is the demonstration that realtime images are obtainable under electrophoretic running conditions with readily available equipment. With further refinement, electric birefringence imaging may be a practical noninvasive and nonnicking technique for monitoring agarose gel electrophoresis on-line. Monitoring may prove useful for controlling and optimizing the field direction sequences used to achieve separations of large nucleic acids. Experiments toward this goal are in progress in our laboratories.

We would like to thank Mr. Miron Drewiacki for his help in preparing the manuscript. This work was supported by NIH grant GM-37006.

\section{REFERENCES}

1. Holzwarth, G., Platt, K. J., McKee, C. B., Whitcomb, R. W. \& Crater, G. D. (1989) Biopolymers 28, 10431058.

2. Crater, G. D., Gregg, R. M. \& Holzwarth, G. (1989) Electrophoresis 10, 310-315.

3. Lumpkin, O. J., Dejardin, P. \& Zimm, B. H. (1985) Biopolymers 24, 1573-1593.

4. Noolandi, J., Slater, G. W., Lim, H. A. \& Viovy, J. L. (1980) Science 243, 1456-1458.

5. Chu, B., Xu, R. \& Wang, Z. (1988) Biopolymers 27, 2005-2009.

6. Sturm, J. \& Weill, G. (1989) Phys. Rev. Let. 62, 14841487.

7. Stellwagen, N. C. (1985) J. Biomol. Struct. Dynam. 3, 299-314.

8. Chu, B., Wang, Z., Xu, R. \& Lalande, M. (1990) Biopolymers 29, 737-750.

9. Akerman, B., Jonsson, M. \& Norden, B. (1985) $J$ Chem. Soc. Chem. Commun. 422-423.

10. Jonsson, M., Ackerman, B. \& Norden, B. ( 1988) Biopolymers 27, 381-414.

11. Akerman, B., Jonsson, M., Norden, B. \& Lalande, M. (1989) Biopolymers 28, 1541-1571.
12. Holzwarth, G., McKee, C. B., Stieger, S. \& Crater, G. (1987) Nucleic Acids Res. 15, 10031-10044.

13. Shick, R. A., Parus, S. J., Matsumura, M. \& Morris, M. D. (1988) Anal. Chem. 60, 1632-1635.

14. Stellwagen, N. C. (1988) Biochemistry 27, 6417-6424.

15. Chu, B., Xu, R. \& Wang, Z. (1990) Macromolecules 23, 790-796.

16. Stellwagen, J. \& Stellwagen, N. C. (1989) Nucleic Acids Res. 17, 1537-1547.

17. Holmes, D. L. \& Stellwagen, N. C. (1989) J. Biomol. Struct. Dynam. 7, 311-327.

18. Pollak, V. A. \& Schulze-Clewing, J. (1988) J. Liq. Chromatogr. 11, 1387-1402.

19. Smith, S. B., Aldridge, P. K. \& Callis, J. B. (1989) Science 243, 203-206.

20. Schwartz, D. C. \& Koval, M. (1989) Nature 338, 520522.

21. Bilhorn, R. B., Epperson, P. M., Sweedler, J. V. \& Denton, M. B. (1987) Appl. Spectrosc. 41, 1114.

22. Smith, S. B. \& Bendich, A. J. (1990) Biopolymers 29, 1167-1173.

23. Ross, P. D. \& Scruggs, R. L. (1964) Biopolymers 2, 231-236.

24. Fredericq, E. \& Houssier, C. (1973) Electric Dichroism and Electric Birefringence, Clarendon Press, Oxford.

25. Kuske, A. \& Robertson, G. (1974) Photoelastic Stress Analysis, John Wiley \& Sons, London.

26. Hunter, R. J. (1989) Foundations of Colloid Science, Vol. I, Clarendon Press, Oxford.

27. Hunter, R. J. (1989) Foundations of Colloid Science, Vol. II, Clarendon Press, Oxford.

28. Everett, D. H. (1988) Basic Principles of Colloid Science, Royal Society of Chemistry, London.

29. The Agarose Monograph (1982) FMC Corporation.

30. Maniatis, T., Fritsch, E. F. \& Sambrook, J. (1982) Molecular Cloning: A Laboratory Manual, Cold Spring Harbor Laboratories, New York.

31. Image Version 1.25 (1990) Rasband, W. NIH Research Services Branch.

32. Houssier, C. \& O'Konski, C. T. (1981) in Molecular Electro-Optics, Krause, S., Ed., Plenum, New York, pp. 309-339.

Received March 12, 1990

Accepted April 22, 1991 